\section{Cahiers de littérature orale}

$66 \mid 2009$

Mémoire des CLO

\title{
« Păcală », ou la fortune de l'absurde
}

\section{Micheline Lebarbier}

\section{OpenEdition}

Journals

Édition électronique

URL : https://journals.openedition.org/clo/732

DOI : $10.4000 /$ clo. 732

ISSN : 2266-1816

Éditeur

INALCO

\section{Édition imprimée}

Date de publication : 1 janvier 2009

Pagination : 147-163

ISBN : 978-2-85831-188-0

ISSN : 0396-891X

\section{Référence électronique}

Micheline Lebarbier, « «Păcală », ou la fortune de l'absurde », Cahiers de littérature orale [En ligne], 66 | 2009, mis en ligne le 25 février 2013, consulté le 01 juillet 2021. URL : http://journals.openedition.org/ clo/732 ; DOI : https://doi.org/10.4000/clo.732

Ce document a été généré automatiquement le 1 juillet 2021.

\section{(c) (†) 8)}

Cahiers de littérature orale est mis à disposition selon les termes de la Licence Creative Commons Attribution - Pas d'Utilisation Commerciale 4.0 International. 


\title{
« Păcală », ou la fortune de l'absurde
}

\author{
Micheline Lebarbier
}

\section{NOTE DE L'ÉDITEUR}

Article paru pour la première fois dans Cahiers de littérature orale, $\mathrm{n}^{\circ}$ 7, 1980, p. 95-113.

(L'auteur a apporté quelques petites modifications à son texte).

1 Păcală est le héros le plus connu de la littérature populaire roumaine. Ses actions peuvent varier d'une région à l'autre, mais le personnage reste immuable. C'est le serviteur qui triomphera de son maitre, le pauvre qui dupera le riche, celui qui saura rétablir l'injustice à son avantage, et ceci par les moyens les plus inattendus et les plus directs. Moyens qu'il serait possible de voir comme étant issus d'une logique de l'absurde.

2 Un résumé du premier conte du Cycle de Păcală sera présenté ci-après. Le Cycle de Păcală est une série de contes facétieux ${ }^{1}$ qui occupent une place très particulière dans la littérature orale roumaine. En premier lieu par la forme des récits: un conte facétieux est, en règle générale, une narration courte constituée d'un seul motif ; or les différents récits du Cycle de Păcală peuvent se rattacher les uns aux autres dans un enchaînement logique.

3 Parmi ces récits, le conte le plus répandu dans la littérature populaire, "Păcală serviteur chez le pope ", est un conte long et chacune des épreuves que le pope impose au héros (et dont ce dernier sort victorieux) peut être considérée comme autant de brefs épisodes².

4 Le Cycle et le personnage de Păcală étant particulièrement caractéristiques du conte facétieux roumain, je propose d'en esquisser ici les lignes principales. Je tenterai de dégager le sens de l'absurde triomphant qui recouvre la plupart des actions du héros ainsi que la fonction que peuvent avoir de tels récits dans la société qui les a émis. Voici résumées deux variantes du premier conte du Cycle, répertorié dans la typologie de 
S.C. Stroescu sous le numéro 3000 . La présentation suit la même dissociation en épisodes, éléments et motifs que celle de S.C.Stroescu, et le même système de numérotation. Ces deux variantes ont été recueillies en novembre 1971, à VădăstriŢa, Olténie, province du sud de la Roumanie auprès de deux narrateurs, tous deux agriculteurs collectivisés, respectivement âgés de 58 ans pour la première variante et de 70 ans pour la deuxième. Dumitru, le narrateur de la première variante, l'avait apprise dans son enfance d'un vieux conteur. Ilie, qui raconta la deuxième, l'avait également entendue d'un vieux conteur. Il l'avait, de plus, lue dans des livres ${ }^{3}$, ce qui pourrait expliquer le fait qu'il est le seul à avoir raconté consécutivement et comme étant un seul conte trois récits complets du Cycle de Păcală.

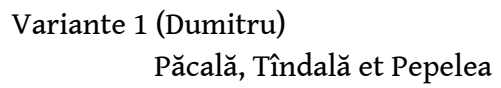

5 Le récit de Dumitru ne s'achève pas là, il enchaîne directement sur un autre texte souvent intitulé « Păcală serviteur chez le pope ", où le héros nanti d'une flûte magique 
entre au service d'un pope. Le contrat entre les deux protagonistes étant : celui qui se fâche aura le nez coupé par l'autre. Il s'en suit une succession de tâches impossibles demandées par le pope et de réponses absurdes données par le serviteur. Il jouera sur les mots en prenant au pied de la lettre les ordres du pope, allant toujours plus loin que n'a voulu aller celui-ci. Enfin, après avoir détruit son bétail et provoqué la mort de sa belle-mère et de sa femme, Păcală provoque celle du pope qui, victime de son avidité, croyant qu'il y a au fond du Danube des fiacres et des chevaux, plonge et se noie.

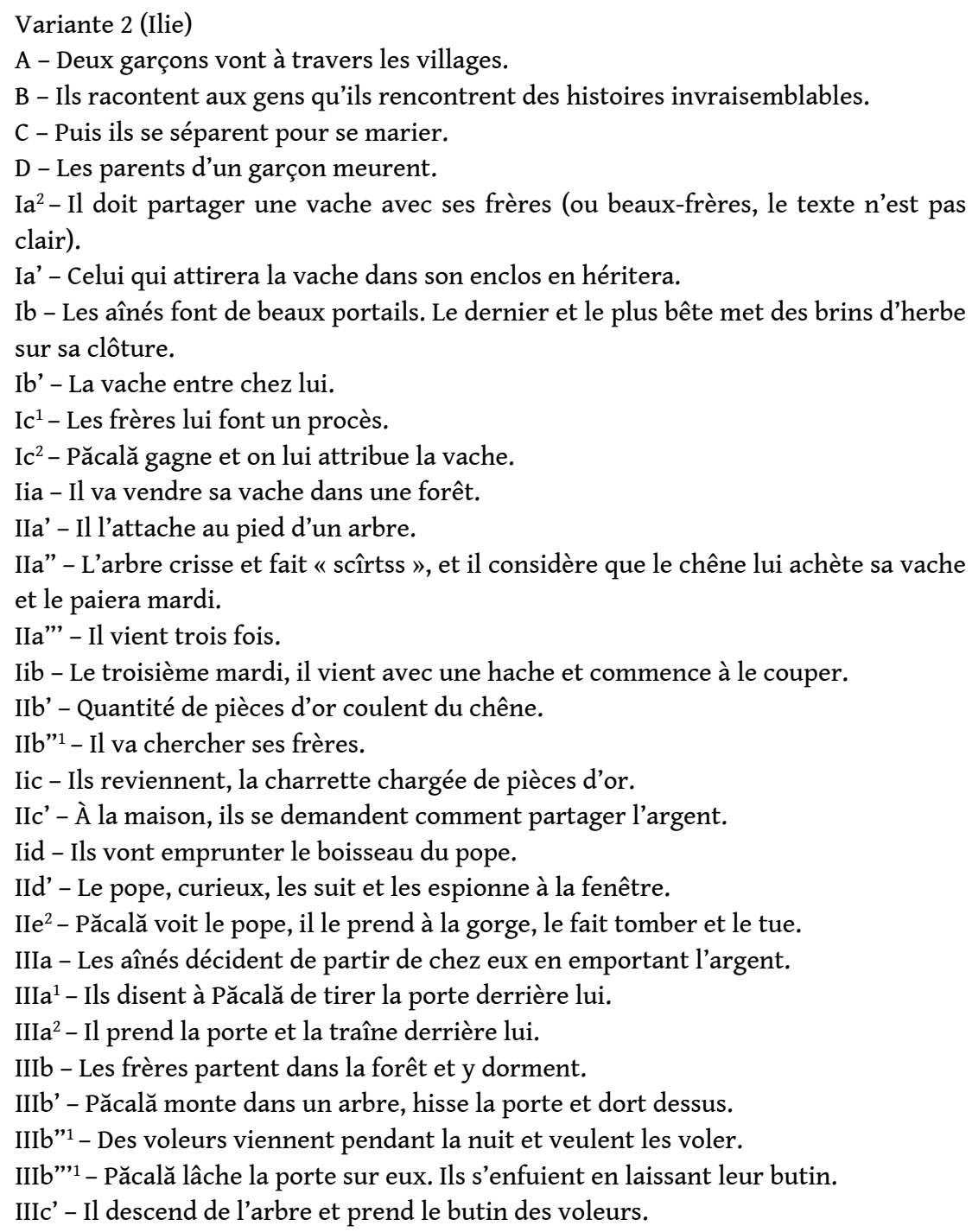

6 Ilie a lui aussi continué avec le conte "Păcală serviteur chez le pope », mais dans cette variante, le pope ne se noie pas. À la fin, il se fâche après que Păcală ait provoqué la mort de sa femme. Selon le contrat initial, il aura le nez coupé ainsi qu'un morceau de peau du dos. Puis le narrateur enchaîne avec un troisième conte où Păcală est encore serviteur, mais chez un mari trompé. Ici le contrat stipule que le héros partira en emportant une charrette et quatre bœufs lorsque les loups hurleront dans l'âtre. Le maitre accepte, sûr qu'une telle situation ne se produira pas. Alors Păcală, par ses facéties, ses à-propos absurdes, traquera les amants coupables pour les montrer finalement à son maître hurlant, ivres et nus, dans la cheminée. On considère, par le truchement de cette métaphore, que le contrat est rempli; le héros emporte la charrette et les bœufs. 
7 Tous ces contes sont répertoriés dans la typologie de S. C. Stroescu et entrent dans le Cycle de Păcală. Il n'existe pas de charnière entre ces différents contes, ce qui s'est passé précédemment n'existe plus dans les contes suivants. Exception faite pour la flûte magique que l'on retrouve dans le deuxième conte et qui est un élément important ${ }^{5}$.

8 Le découpage de ce conte a été établi d'après la typologie de S. C. Stroescu 6 . J'ai respecté son analyse structurale et son système de numérotation. Les chiffres romains indiquent la dissociation en épisodes, et aux petites lettres de l'alphabet correspondent les éléments du cadre des épisodes (Stroescu, 1969, XI). En revanche, les lettres portant un exposant (par exemple $\mathrm{Ia}^{1}, \mathrm{Ia}^{2}$ ) signalent des variations par rapport au conte type, ces variations étant également répertoriées dans la Typologie de référence. Les signes a', $a ", b$, b", indiquent que les résumés présentés ici sont plus riches en éléments que ceux donnés dans la Typologie de S. C. Stroescu, tout en correspondant à son découpage.

9 Les séquences $\mathrm{A}, \mathrm{B}, \mathrm{C}, \mathrm{D}$, de la variante 2 sont à mettre à part. Elles résultent d'un amalgame avec un autre conte du Cycle de Păcală (3011). Ainsi, les deux variantes ont en commun, par rapport au conte-type $3000^{7}$, les épisodes suivants : Ia, Ib, IIa, IIb, IIIa, IIIb.

\section{ÉTUDE}

\section{LE RÉCIT}

10 Ce premier conte du Cycle de Păcală se présente comme une introduction. Le héros est situé dans son contexte familial où déjà il est différent de ses frères (Ia). Les deux narrateurs insistent sur cette différence: «Celui qu'on tenait pour le plus petit et le plus bête... » Ilie, variante 2 , et Dumitru dans la première variante va jusqu'à préciser : «Le dernier frère était débile ». La différence entre Păcală et les " autres » est posée dès le départ, ici entre ses frères et lui et dans d'autres contes, entre son environnement social et lui (son maître, le pope, le village...).

11 Il est le plus jeune d'une famille de trois frères. Ce trait est immuable, il existe dans tous les récits où Păcală est le héros, qu'il s'agisse du corpus que j'ai recueilli, des pièces de littérature orale présentées par 0 . Bîrlea ${ }^{8}$, ou de celles qu'a répertoriées S. C. Stroescu (1969). En règle générale, le dernier frère a un statut particulier, il se situe entre le monde de l'enfance et celui des adultes, à mi-chemin du monde féminin et du monde masculin.

... la nature androgyne de l'enfant ou des jeunes gens ne pourrait-elle pas expliquer le fait que c'est toujours (ou presque) le plus jeune des frères ou des sœurs (...) qui dans les contes est le plus beau, le plus intelligent et le plus fort? Il jouit des possibilités des deux sexes.

(Popova, 1970, 118, note 17)

Si Păcală est explicitement déclaré débile ou bête par les deux narrateurs, la suite de ce conte (et de la majorité des contes du Cycle) montrera que cette "bêtise » ou cette " débilité » est en réalité une force. Les actes en apparence les plus absurdes du héros ont toujours un résultat positif, et il y aurait lieu de se demander s'ils ne recouvrent pas une logique inversée. La possession de la vache nécessite une épreuve dont le garçon sort victorieux (Ib, Ib'). L'enclos de feuillage correspond à une image de la maison. La "maison » des animaux doit être adaptée aux besoins des animaux et non à ceux de l'homme. Păcală a correctement réagi, à l'inverse de ses frères qui ont construit des 
«maisons» pour les animaux conformes aux besoins et aux goûts de l'homme. D'ailleurs, dans la variante 2 son succès sera confirmé par les autorités $\left(\mathrm{IIc}^{2}\right)$. Dumitru (variante 1) précise que pour le petit, il n'est plus resté de matériaux pour construire son enclos, aussi a-t-il fait un enclos de feuillage. Ce trait, s'il insiste sur la coalition des aînés envers le plus jeune, soulève aussi une question : cela pourrait-il signifier que si les matériaux avaient été suffisants, ou si les frères avaient accepté de partager avec lui, Păcală aurait-il mal passé l'épreuve ? Il ne semblerait pas. Dans l'autre variante, il fait spontanément un enclos de feuillage, ainsi que dans la Typologie de S. C. Stroescu. Aussi peut-on en déduire qu'il s'agit d'un détail donné par le narrateur qui a (peut-être) voulu mettre en valeur l'égoïsme des frères et le rejet de leur benjamin. Leur jalousie confirme la particularité de celui-ci. Et le juge pourrait également signifier la reconnaissance par les autorités de sa supériorité.

Un autre acte apparemment absurde de Păcală est le départ en forêt, de même que la vente de la vache à un arbre (IIa"). Pourrait-on, en prenant comme résultat le trésor de l'arbre 9 , voir ce départ comme un voyage de type initiatique? La recherche d'un acheteur, donc d'argent, la traversée en forêt, pourraient y faire penser, ainsi que le dialogue avec l'arbre. Mais dans la forêt, le héros ne rencontre pas d'épreuves "concluantes" sur son parcours. En revanche, il semblerait, du moins dans la variante 1, qu'il suive au pied de la lettre les plaisanteries des gens rencontrés en chemin (IIa $\left.{ }^{1}\right)$; ce qui conduit Păcală à mutiler sa vache et à provoquer sa mort (IIa'1). Ou bien pourrait-on y voir, au contraire, une épreuve ? L'épreuve étant alors de refuser une apparente logique. Ce trait se retrouve dans d'autres contes facétieux comme une illustration de l'absurde, et alors le héros ne récolte rien, tant s'en faut ! Ici, l'absurde conduit Păcală à un arbre au trésor ${ }^{10} \ldots$

Alors de quel absurde s'agirait-il ? D'un absurde apparent, le sens profond des choses étant à ce point caché qu'il faut les inverser pour obtenir un résultat ? La «bonne voie » est-elle celle de tout le monde, celle du "bon sens", ou bien faut-il faire exactement l'inverse ? Suivre les conseils aberrants d'inconnus pour découvrir un trésor, exécuter si scrupuleusement des ordres extravagants qu'ils se retournent contre celui qui les a donnés (comme dans le conte " Păcală serviteur chez le pope », mentionné plus haut)? Peut-être serait-ce une des fonctions du Cycle de Păcală, une fois dépassé le côté distrayant de ces contes, que d'amener leurs auditeurs à se poser ces questions.

Ainsi en roumain «mardi » se dit marŢi, en transcription /marts/, et l'onomatopée peut être confondue avec le crissement des branches de l'arbre (IIa"). D'autre part, le mardi est reconnu dans la tradition populaire roumaine comme étant le jour où les entités surnaturelles ont le plus de pouvoir. Ce sera le jour propice à divers exercices magiques, charmes, incantations... Par exemple, c'est de préférence le mardi qu'auront lieu les veillées de jeunes filles pour faire venir les garçons par des pratiques incantatoires, donc des veillées où les pratiques magiques ont largement leur place (Alvarez-Pereyre, 1976, 41). Ainsi un conte recueilli en décembre 1971 auprès d'une narratrice de Breb (Maramureș, Nord de la Roumanie), parle d'une veillée de jeunes filles où, à la place des garçons, ce sont les diables qui viennent ${ }^{11}$. Ils déchiquettent une jeune fille qui ne s'est pas enfuie et suspendent ses intestins aux clous qui supportent les ustensiles de cuisine. Cette veillée s'était tenue un mardi comme le montrent ces vers qui émaillent le conte : 


\begin{tabular}{|l|l|}
\hline $\begin{array}{l}\text { De marŢi sara în șezătoare } \\
\text { MaŢele-s pe cuișoare }\end{array}$ & $\begin{array}{l}\text { Depuis mardi soir à la veillée } \\
\text { Les intestins sont sur les clous }\end{array}$ \\
Tăt măŢucu și cuiuŢu & Tout boyau a son clou \\
Tăt cuiuŢu și măŢucu & Tout clou a son boyau
\end{tabular}

16 Ainsi le mardi est un jour spécial, c'est un jour où l'on peut s'attendre à ce qu'il se passe des choses inhabituelles. De plus, le fait que le héros doive revenir trois fois (IIa"') avant de découvrir le trésor peut être vu comme un élément s'ajoutant au côté particulier du mardi. Le chiffre trois a un sens d'achèvement qui se retrouve dans beaucoup de rites. Par exemple, le jour des noces, les jeunes mariés doivent faire trois fois le tour de l'église, puis de la maison, avant d'y entrer. La jeune mariée doit refuser trois fois la coiffure de femme mariée avant de l'accepter des mains de son parrain. Le mois le plus propice aux incantations et envoûtements magiques est le mois de mars, troisième mois de l'année. Les exemples pourraient être multipliés à l'infini. Donc, le mardi, le chiffre trois (n'oublions pas que Păcală est le troisième frère) ont un sens symbolique très marqué que le héros est conduit à redécouvrir et à utiliser à son avantage.

17 Cependant, un élément viendrait contrebalancer cette hypothèse : le trésor de l'arbre comme résultat d'une quête réussie. L'attitude du garçon devant le trésor n'est pas celle d'un individu ayant entièrement acquis son autonomie, il part chercher ses frères pour qu'ils l'aident (IIb", IIb"1). Il n'aurait donc pas la connaissance et la maturité nécessaires, puisqu'il ne considère pas ces richesses comme les siennes, comme étant le résultat d'une quête autre que celle de la vente de la vache. Dans la première variante, il prend ce qu'il croit être le prix de sa vache (IIb") et laisse le reste, que ses frères et lui viendront chercher ensuite. Dans la deuxième variante, il va directement chercher ses frères sans prendre quoi que ce soit (IIb"1). Le trésor de l'arbre est partagé entre les trois frères et ne revient pas à Păcală seul, ce qui serait le cas s'il s'agissait du fruit d'un voyage à caractère initiatique. Cependant, l'arbre peut être considéré comme le médiateur, le substitut végétal de la mère nourricière (Calame-Griaule, 1969b, 55), mais pas comme l'arbre initiatique.

18 Pourrait-on y voir, alors, une parodie d'initiation? Le trésor est obtenu par le «meurtre » de l'arbre, le partage du butin entraîne indirectement le meurtre du pope $\left(\mathrm{IIe}^{1}, \mathrm{IIe}^{2}\right)$. La mort du pope entraîne un deuxième départ dans la forêt, collectif, cette fois ; autres signes que le premier départ n'était pas un bon départ.

19 Le personnage du pope est un des plus importants des contes facétieux roumains. Il est présent dans la majorité de ces récits où il tient très souvent le rôle de l'antagoniste. Ainsi un tiers de notre corpus ${ }^{12}$ met en scène le pope dans des situations négatives. Il sera tourné en dérision pendant l'exercice de ses fonctions (pendant qu'il officiera, par le Tsigane qui se confesse...), ou bien libertin châtié et ridiculisé par le mari de la femme convoitée, ou mari trompé et ignorant son infortune, ou encore maître despotique et roulé par son serviteur (voir encore le conte « Păcală serviteur chez le pope » déjà mentionné). En fait, le pope ne sera jamais vu comme un habitant du village, mais comme l'homme d'Église, représentant de la morale et de la religion. C'est pourquoi dans les contes facétieux, il sera puni plus souvent que d'autres de transgresser les lois qu'il représente ${ }^{13}$. C'est un des personnages riches du village, c'est donc à lui que l'on va emprunter ; étant riche il est susceptible d'avoir un instrument de 
mesure, ayant assez de biens pour avoir besoin de les mesurer (IId). (Dans les deux variantes le pope s'étonne que des garçons pauvres aient besoin d'un boisseau.) Avide et curieux, il épie les frères (IId') ; maladroit, il se fait découvrir ; malchanceux, il se fait tuer. Dans la variante 1, sa dépouille mortelle est tournée en dérision; pendu par la barbe (symbole de son appartenance à l'église) à un pommier (IIg), il fait l'objet d'un quiproquo, où le voisin lui plantant sa fourche dans le corps croit l'avoir tué (IIg'). La dérision existe et dans la vie et dans la mort : dans la vie, par son comportement ; dans la mort, par ce qui est fait de son cadavre.

La résolution d'un conflit nécessite la mort, c'est un trait fréquent dans cette littérature populaire. L'évolution d'une situation n'est possible que par la mort d'un des protagonistes. Ici, la mort du pope permet le passage à un autre type de situation : il ne sera plus question du trésor récolté, mais d'un deuxième départ dans la forêt (IIIa). Cette fois, les trois frères partent ensemble et Păcală se distingue encore de ses frères en emportant un objet de leur maison (IIIa ${ }^{2}$ - la maison, lieu où l'on est à l'abri - qui deviendra un instrument de défense. La porte «tirée " par Păcală correspond à un jeu de mots en roumain qui a son équivalent en français (IIIa $\left.{ }^{1}\right)$, « tire la porte derrière toi », et il l'emporte. Cette porte qu'il jette sur les voleurs (IIIb"'1) lui permet de les mettre en fuite et de s'emparer de leur butin (IIIc'), alors que ses frères seront maltraités. La montée sur l'arbre symbolise la rupture avec eux (IIIb') ${ }^{14}$. Le rôle protecteur de l'arbre ressort une nouvelle fois ${ }^{15}$. Dans la première variante, un marchand d'encens remplace les voleurs (IIIb"), si bien qu'on ne peut pas tirer de conclusions moralisatrices comme on aurait pu être tenté de le faire avec ce seul motif. Les voleurs sont menaçants, le marchand d'encens ne l'est pas, mais ils subissent le même sort. Par l'intermédiaire des premiers, le héros obtient un bien terrestre dont il ne sera plus question ailleurs. Par l'intermédiaire du second, il obtient de Dieu une flûte magique (IIId $\left.{ }^{2}\right)$, qui aura un rôle important dans les contes suivants. Le seul parallèle à établir entre le marchand et les voleurs, est qu'il y a quelque chose à leur prendre. Dans la Typologie de S. C. Stroescu, il est question également de trois marchands, du sac d'encens ${ }^{16}$ et d'une cornemuse magique. On peut donc penser que le motif initial a été contaminé avec d'autres motifs. Cependant, la flûte semble être un instrument de musique plus spécifique de la société traditionnelle roumaine que la cornemuse. Le berger (ou le pâtre) aura souvent une flûte qu'il aura taillée et travaillée lui-même, et dont il jouera pour meubler sa solitude ${ }^{17}$.

21 Ce qui est à noter dans ce conte, c'est la différence toujours vérifiée de Păcală vis-à-vis de ses frères. Dans le dernier épisode sa rupture avec eux, symbolisée par sa montée sur l'arbre, est confirmée par le don que Dieu lui fait d'un objet magique, qui se révélera être un objet de pouvoir.

Ce conte est donné par les narrateurs - et dans la typologie de S.C. Stroescu - comme étant le début du premier conte-type du Cycle de Păcălă. Il peut apparaître comme une présentation du héros, où ses caractéristiques particulières sont esquissées pour prendre plus de force dans les contes suivants. Sa victoire sur ses rivaux directs, ses frères, l'ont préparé à un autre affrontement plus fondamental (qui sera étudié ultérieurement). Il emporte, comme résultat de cette première victoire, une flûte magique devant lui assurer la suprématie sur son adversaire dans le conte «Păcală serviteur chez le pope », lequel fait suite à celui-ci. Il n'arrivera pas devant le pope démuni comme ses frères, mais riche d'avoir réussi une première épreuve et porteur d'une flûte enchantée. 
23 À défaut de pouvoir présenter le Cycle complet, ce premier récit nous permet de pressentir le personnage de Păcală et d'évaluer son importance dans la littérature populaire roumaine.

\section{LE PERSONNAge de PĂCALĂ}

$$
\text { duper ». Păcală est donc par définition «celui qui joue des tours ». Seulement, aucun }
$$
tour n'est innocent : l'individu qui est joué est celui qui :

- enfreint les règles sociales (la femme infidèle, le mauvais maître),

- est en haut de l'échelle sociale (le pope, les notables),

- est suffisamment sot pour se laisser duper (par crédulité ou avarice).

Ces trois catégories pouvant successivement abriter le même personnage : le pope, mauvais maitre, qui saute dans le Danube croyant y trouver un fiacre et des chevaux (comme dans le récit qui suit la variante 1).

D'une part, le Cycle de Păcală est le reflet d'une très féroce critique sociale. Il est, par le biais même de ses bouffonneries, le redresseur de torts, le vengeur du peuple ; le rire permet de se libérer pendant le temps du récit des contraintes réelles de la société. G. Mendel écrit: «Toute activité ou récit à contenu fantasmatique intervient comme réponse à une frustration ${ }^{18}$.» $(1968,64)$. Peut-être y aurait-il également lieu de se demander si l'on n'investit pas le héros des actes que l'on aimerait être soi-même capable de faire. Si l'on ne dénoue pas par le truchement de ce personnage des nœuds qui ne peuvent être dénoués que dans la scène imaginaire. C'est vraisemblablement la raison principale d'une telle démesure, et si la démesure, le fantasme, est proportionnel à la frustration, l'on peut évaluer celle que supporte ou a supporté la société productrice de ces récits.

D'autre part, l'analyse de cette figure essentielle du conte facétieux roumain ne peut contester une certaine parenté avec d'autres personnages du même type, issus d'autres sociétés, chaque pays ayant, en fonction de ses caractéristiques propres, adapté son héros ${ }^{19}$.

Ainsi Till Ulespiègle ${ }^{20}$ qui fait son apparition dans l'édition allemande dès 1483 , et présente avec Păcală ces traits communs : prendre au pied de la lettre tout ce qu'on lui dit, faire selon les paroles et non selon l'intention, montrer aux autres leur bêtise... Mais contrairement au Cycle de Păcală, le curé rira des facéties de Till et n'en sera pas forcément la victime, la femme infidèle ne sera pas démasquée et châtiée, elle donnera simplement plus à la quête pour que les autres ne sachent pas qu'elle pèche. Păcală en comparaison est un personnage plus "moral », présentant un côté plus accentué de redresseur de torts. Les cibles de Păcală sont beaucoup plus sélectives, ce sera :

- le pope, avide et curieux, mauvais maître par excellence ;

- les voleurs qui verront leur butin dérobé ;

- la femme infidèle, violemment corrigée par son mari ;

- enfin... tous ceux qui enfreignent les lois établies.

Ainsi Nasredin Hoca, personnage célèbre des contes facétieux turcs, qui fait son apparition dans la littérature du seizième siècle, date à laquelle on commence à compiler les récits de ses facéties (Boratav, 1971) ; mais c'est à partir de 1850 que 
paraissent des recueils qui lui sont consacrés. P. Boratav estime à six les catégories thématiques de ces anecdotes :

-1) niaiserie de Nasredin, réelle ou feinte ;

-2) anecdotes licencieuses ;

-3) Nasredin représentant de la sagesse populaire (combat contre la violence, la tyrannie, et autres expressions de critique sociale);

-4) situations comiques de tout genre ;

-5) malices et fourberies ;

-6) Nasredin, personnage de légende vénéré comme un saint.

Il est clair que les facéties de Păcală peuvent s'inscrire dans ces catégories, réserves faites peut-être pour la sixième. Păcală est, certes, un personnage célèbre sinon un héros de légende, mais il n'est pas ressorti, du moins des différents entretiens avec les narrateurs, qu'il était vénéré comme un saint. Il reste cependant que la question d'une certaine contamination se pose et l'auteur roumain Anton Pann aurait largement contribué au rayonnement de la tradition nasredinienne en Roumanie et dans les Balkans ${ }^{21}$. Certains auteurs comme Petre Ispirescu ou Teodor Speranţa auraient utilisé des thèmes facétieux provenant partiellement de cette tradition ${ }^{22}$. Mais Nasredin ayant surtout rayonné dans la littérature écrite, il est très difficile d'évaluer avec exactitude dans quelle mesure certains motifs oraux ont été contaminés par des motifs écrits.

La même difficulté existe inversement : cette littérature folklorique écrite provient de sources orales qui auront par la suite été consignées par écrit. Un des narrateurs interrogés dit avoir entendu dans son enfance le conte que l'on venait d'enregistrer et l'avoir lu plus tard dans un livre. Cette fluctuation se retrouve dans toutes les sociétés ayant une tradition orale et une écriture ${ }^{23}$.

Cependant dans la culture populaire roumaine, la consignation par écrit remontant seulement à la seconde moitié du XIX siècle, on peut accréditer l'hypothèse d'une authenticité orale certaine. Quant à la contamination du personnage (sans oublier que la Roumanie a été sous protectorat ottoman pendant plusieurs siècles et sans négliger la circulation des manuscrits), il serait nécessaire de confronter chaque type de récit avec des variantes provenant de chaque tradition afin d'établir ce qui appartient à qui. Par ailleurs, il est certain que les mêmes motifs circulent à travers l'Europe et particulièrement l'Europe balkanique. Ces motifs paraissant semblables dans les différentes sociétés, elles ont secrété des personnages aux caractéristiques proches et ayant la même fonction cathartique, chacune le modelant au gré de ses nécessités profondes.

En conclusion, quelles seraient les significations à un niveau plus complexe de la permanence d'un tel personnage? Les réponses des narrateurs lors de l'enquête sur la réalité de Păcală étaient : «Păcală, il vit dans le village d'à côté », ou bien « Il vivait il y a trente, quarante ans, je ne sais pas s'il vit encore ». Et cet autre : « Păcală, c'est un peu chacun de nous ». Păcală, tout en conservant un aspect irréel est proche, et le dernier interlocuteur cité a peut-être le mieux compris le rôle que sa société assignait à celuici : une certaine identification de chacun avec le personnage, afin de réaliser pendant le temps du conte ce qu'il ne peut se permettre dans la réalité. Au-delà d'une virulente critique sociale, cette escalade de l'absurde a vraisemblablement une fonction 
libératrice. Mais ne pourrait-on pas y voir plus encore? Ne pourrait-on, pour ce héros européen émettre comme hypothèse ce que G. Calame-Griaule avance dans sa conclusion d'Histoires d'Enfants Terribles (1980, 247-249): la démesure du personnage asocial est le fait d'un initié, d'un individu détenteur de la connaissance ? À un moindre degré, certes, l'initiation étant dans les sociétés européennes un pâle souvenir de ce qu'elle est encore dans les sociétés africaines. Cependant, ne pourrait-on pas voir dans ce type de conte une survivance d'un message qu'il faudrait savoir décoder une fois posées certaines données? Le triomphe de ce qui est absurde en apparence, le meurtre de ceux qui détiennent un pouvoir social qui n'est pas le vrai pouvoir, la destruction ou l'abandon de richesses qui masquent d'autres richesses, la prescience constante que le héros manifeste pour déjouer tous les pièges et prévenir toutes les embûches... (que le terme de năzdrăvan appliqué à Păcală vient confirmer). Ceci pourrait aider à mieux cerner ce personnage fascinant et si complexe.

\section{BIBLIOGRAPHIE}

AlVAREZ-PEREYRE, Frank, 1976, Contes et tradition orale en Roumanie (La fonction pédagogique du conte populaire en Roumanie), Paris, SELAF.

BîRLEA, Ovidiu, 1966, Antologie de proză populară epică (Anthologie de prose populaire épique), București, Editură pentru literatură, 3 vol.

BoratAV, Perter, 1971, Les aires d'expansion des anecdotes de Nasredin Hoca, Communication au $1^{\mathrm{er}}$ congrès International des Études balkaniques et Sud-Est européennes, Sofia.

CALAmE-Griaule, Geneviève, 1969a, Introduction, in G. Calame Griaule (éd.), Le thème de l'arbre dans les contes africains, Paris, SELAF, p. 19-24.

CALAME-GRIAUle, Geneviève, 1969b, L'arbre au trésor, in G. Calame Griaule (éd.), Le thème de l'arbre dans les contes africains, Paris, SELAF, p. 25-58.

Calame-Griaule, Geneviève, 1980, Conclusion, in V. Görög-Karady, S. Platiel, D. Rey-Hulman, C. Seydou, Histoires d'Enfants Terribles (Afrique Noire). Études et Anthologie, Paris, Maisonneuve et Larose, p. 239-250.

GöRÖG-KARADY, Veronika, Platiel, Suzanne, REY-Hulman, Diana, SEYDOU, Christiane, 1980, Histoires d'Enfants Terribles (Afrique Noire). Études et Anthologie, Préface et conclusion par G. Calame-Griaule, Paris, Maisonneuve et Larose.

JANNET, P. (Commentaires de), 1866, Les aventures de Till Ulespiègle, $1^{\text {re }}$ traduction complète faite sur original allemand (1519), précédée d'une notice et suivie de notes, Paris, Picard.

LEBARBIER, Micheline, 1976, Les notables libertins, Cahiers de Littérature orale, $\mathrm{n}^{\circ} 1$, Paris, POF, p. 135-156.

MENDEL, Gérard, 1968, La révolte contre le père. Une introduction à la sociopsychanalyse, Paris, Payot.

PANN, Anton, 1961, Fabule și istorioare. Nezdrăvăniile lui Nastratin Hogea (Fables et histoires. Les facéties de Nastratin Hogea), București, Editura pentru literatură (1 ${ }^{\text {re }}$ éd., 1852). 
PAULME, Denise, 1976, La mère dévorante. Essai sur la morphologie des contes africains, Paris, Gallimard.

Popova, Assia, 1970, L'arbre et le mariage, in G. Calame Griaule (éd.), Le thème de l'arbre dans les contes africains II, Paris, SELAF, p. 63-118.

STROESCU, Sabina Cornelia, 1969, La typologie bibliographique des facéties roumaines, Bucarest, Éditions de l'Académie de la République socialiste de Roumanie, 2 vol.

\section{NOTES}

1. Laquelle est particulièrement riche et a fait l'objet d'un chapitre qui lui est entièrement consacré dans La typologie bibliographique des facéties roumaines publiée par Sabina Cornelia Stroescu en 1969. Quatre-vingt-cinq facéties-types y ont été recensées, sans compter leurs nombreuses variantes.

2. Je reprends ici la terminologie utilisée par S. C. Stroescu dans son introduction (1969, XI, note 6) : «Épisode dans le sens général de fragment d'action. L'Épisode est formé de motifs groupés en entité parfaite. Les Épisodes ont une forme fixe et, tout en les gardant intacts on peut les assembler dans de diverses combinaisons, dans un enchaînement logique, ce qui donne naissance à des variantes du même type de narration, ou à des types différents. (...) » "L'élément représente la plus petite partie constitutive d'une narration » (id., note 7). « Le motif représente une réunion d'éléments dans une relation qui lui donne un certain contenu... » (ibid., note 8).

3. Les récits oraux, et particulièrement les contes facétieux, ont fait l'objet au XIX siècle et au début $\mathrm{du} \mathrm{xx}^{\mathrm{e}}$ de publications par les écrivains et les érudits locaux qui les recueillaient, les transcrivaient et les publiaient dans les gazettes régionales ou dans des recueils. Certains écrivains, plus contemporains, ont puisé aux sources orales et ont publié des ouvrages signés de la formule sub îngrire, textuellement "sous le soin de ». Grâce à ce travail, une partie de cette tradition avait pu au moment de l'enquête garder une continuité.

4. Ancienne mesure de capacité pour les céréales, équivalente en Roumanie à 20 ocale (un oca, selon les régions, pouvait contenir entre 1288 et 1520 millilitres).

5. Plusieurs motifs du Cycle de Păcală sont attestés chez Aarne et Thompson, A.Th. 559, 3458, 3696.

6. La typologie de S.C. Stroescu a été établie à partir de 169 recueils, 341 périodiques et de nombreux autres manuscrits et publications provenant de toutes les régions de Roumanie. Elle a également enrichi sa documentation par des enquêtes sur le terrain de 1950 à 1969.

7. La numérotation des contes facétieux part du chiffre 3000. Ce conte correspond aux trois premiers épisodes de cette première facétie-type qui en comporte douze dans la Typologie.

8. Ovidiu Bîrlea (1966). Son anthologie en trois volumes a été établie d'après des enregistrements effectués par des chercheurs de l'Institut d'Ethnographie et de Folklore de Bucarest de 1950 à 1962. L'auteur a minutieusement transcrit et redonné intégralement le texte oral en tenant compte des variations de prononciation, en notant les gestes des narrateurs...

9. "... les biens de l'arbre sont un véritable "trésor", celui de la connaissance, que le héros conquiert au cours d'une quête initiatique... » (Calame-Griaule, 1969a, 21).

10. Dans d'autres contes du Cycle, l'absurde, l'observation au pied de la lettre d'injonctions saugrenues conduiront Păcală à déjouer les pièges de ses adversaires et à sortir victorieux de toutes les situations.

11. F. Alvarez-Pereyre $(1976,98)$ rapproche dans son étude les diables des garçons : « Il semble possible de dire que les diables sont, symboliquement, l'équivalent des garçons... Dans l'une des variantes la conteuse juxtapose les termes de diables et de garçons ». 
12. Recueilli dans trois villages au cours de l'automne et de l'hiver 1971-1972: VădăstriŢa (Olténie), Siliștea Snagov (à une cinquantaine de kilomètres de Bucarest), Breb (dans le Maramureș).

13. Pour une étude plus détaillée d'un des aspects du comportement du pope, le libertinage, voir Lebarbier (1976).

14. Dans le récit qui fait suite à celui-ci, les aînés s'engagent chez un pope et seront affamés, exploités et mutilés. Păcală, en revanche, sortira victorieux de toutes les épreuves imposées par ce mauvais maître.

15. Ce motif des voleurs sous l'arbre est attesté chez Aarne et Thompson (type 1653) et se retrouve dans beaucoup d'autres contes.

16. L'odeur de l'encens qui monte agréablement jusqu'à Dieu: pourrait-on voir dans ce motif une lointaine réminiscence biblique? Noé, après avoir été sauvé du déluge, offre à Dieu un holocauste et il monte jusqu'à lui une "agréable odeur ». Si tel était le cas, le côté privilégié de Păcală en serait encore renforcé.

17. Un des narrateurs de VădăstriŢa, ancien berger, était fier de montrer sa collection de flûtes qu'il avait fabriquées lui-même et dont il jouait fort bien. Il y en avait de deux sortes : celles de taille assez petite (30 à 40 centimètres), qui produisaient des sons assez aigus (fluier), et d'autres beaucoup plus longues (environ un mètre), d'un diamètre plus large et qui donnaient de beaux sons graves (caval).

18. Ce thème est attesté ailleurs, notamment en Afrique. Voir Paulme (1976, chapitre III «Le mâle qui met bas » et particulièrement le personnage de l'Enfant Malin, p. 224-225).

19. L'Enfant Malin d'Afrique (Paulme, 1976) vient d'être cité. Sans vouloir se hasarder à faire une comparaison hâtive entre notre héros et ceux des contes africains, signalons cependant l'étude que Görög-Karady, Platiel, Rey-Hulman, Seydou (1980) ont consacrée à l'Enfant Terrible d'Afrique noire. Notre personnage présente un certain nombre de traits communs avec ce héros africain, et dans une moindre mesure avec l'Enfant Malin (hiérarchie sociale tournée en dérision, renversement par l'absurde de certaines situations, le héros utilisant à son avantage les moyens employés par son adversaire...).

20. P. Jannet (commentaires de), 1866, voir sa page IX.

21. A. Pann, 1852 ( $1^{\text {re }}$ éd.). Le terme de nezdrăvăniile se retrouve également appliqué à Păcală (Năzdrăvăniile lui Păcală, Omul năzdrăvăn...). Ce terme a le sens de "facéties, farces ", mais également celui de " puissance surnaturelle ». Cependant si, d'après les textes d'Anton Pann, les cibles de Nasredin sont les mêmes que celles de Păcală (les riches, ceux qui méprisent les paysans et n'ont pas leur sagesse...), elles sont là aussi moins sélectives et le rire est plus indulgent. L'ironie suffit à punir celui que l'on vise et la démesure semble moins importante.

22. P. Boratav cite pour ces informations Gh. I. Constantin (1967, « Der Islam », XLIII).

23. Cette question ne se pose pas dans les civilisations à tradition orale, africaines par exemple.

\section{RÉSUMÉS}

Le personnage de Păcală est le héros privilégié des contes facétieux roumains. Un Cycle de récits décrit ses exploits à contre-courant, ses à-propos absurdes, les tours qu'il joue à ceux qui transgressent les lois ou qui veulent s'en prendre à lui. Deux variantes des premiers épisodes du premier conte du Cycle sont présentées ici. Elles ont été enregistrées en automne 1971 auprès de 
deux narrateurs d'un village d'Olténie. L'étude - s'appuyant sur les différents textes du corpus recueilli, l'enquête auprès des narrateurs ainsi que sur La typologie bibliographique des facéties roumaines de Sabina Cornelia Stroescu -, démontrera que ce premier conte apparaît comme une forme d'introduction aux caractéristiques si particulières de ce personnage. Il n'est d'ailleurs pas sans présenter divers traits communs à d'autres figures facétieuses de la littérature populaire (orale et écrite) qui a circulé en Europe et dans les Balkans.

The character Păcală is the central hero of comic tales in Romania. A cycle of tales describes his contrary deeds, absurd remarks, and practical jokes against those who transgress his laws or want to attack him. Two variants of the first episodes of the first tale in the cycle are presented here. They were recorded fall 1971 from two narrators in a village of Oltenia. This analysis is based on different texts of the collected corpus, discussions with narrators, and the bibliographical typology of Romanian jokes and anecdotes by Sabina Cornelia Stroescu. It demonstrates that the first tale can be seen as an introduction to the very particular character traits of this hero. In fact, he shares many common traits with other comic heroes in the popular literature (oral and written) that circulated in Europe and the Balkans.

INDEX

Thèmes : anthropologie (Europe)

Mots-clés : contes facétieux, héros facétieux, absurde, critique sociale, initiation

Index géographique : Roumanie

Keywords : Rumania, Comic Tales, Comic Hero, Absurd, Social Critique, Initiation, Anthropology 